\title{
STUDIE
}

\section{Hohe Wertschöpfung}

\author{
Walter Häcker und Doris Knaier
}

Ein Gutachten der Katholischen Stiftungsfachhochschule München für das Bayerische Sozialministerium zeigt den ökonomischen und sozialen Wert Bürgerschaftlichen Engagements: Ohne Freiwillige würden die Kosten in vielen Einrichtungen explodieren, viele benachteiligte Menschen würden eine glaubwürdige Lobby verlieren und das soziale Klima würde kalt und rau.

Bürgerschaftliches Engagement schafft selbstverständlich auch Geldwert, doch begründen Menschen den Wert ihrer freiwilligen Arbeit, ihres Engagements ganz anders: Sie wollen gesellschaftliche Werte nach ihren Vorstellungen fördern. Sie engagieren sich besonders gerne, wenn

Katholische Stiftungsfachhochschule München damit, ein »Gutachten zum Wert des Bürgerschaftliche Engagements in Bayern « anzufertigen. Projektleiter war Prof. Dr. Gerhard Kral vom Institut für Fort- und Weiterbildung, Forschung und Entwicklung der Stiftungsfachhochschule. Um die soziale und ökonomische Bedeutung des Bürgerschaftlichen Engagements zu belegen und auf kommunaler Ebene effektiver fördern zu können, war es dem Ministerium wichtig, ein aussagekräftiges Bild darüber zu gewinnen, welche Werte - ökonomische und soziale durch die freiwillig engagierten Bürgerinnen und Bürger geschaffen werden.

Die ausgewählten Untersuchungsgebiete unterscheiden sich stark: der ehemals sehr ländliche, grenznahe, derzeit wirtschaftlich aufstrebende Landkreis Cham/Oberpfalz und die Universitäts-

\section{»Jeder Euro für Engagement-Förderung erbringt eine Wertschöpfung von sechs bis sieben Euro"}

sie damit etwas für andere oder die Allgemeinheit schaffen können - etwas, das es ohne ihre Mitarbeit so nicht gäbe.

Den Blick gleichermaßen auf den ökonomischen Nutzen wie auch auf die sozialen Wirkungen zu richten, war der Auftrag eines Gutachtens für das Bayerische Sozialministerium. Die Ergebnisse zeigen gute Gründe und Möglichkeiten auf, Bürgerschaftliches Engagement noch attraktiver zu gestalten.

Die Bereitschaft zur Mitarbeit steigt, wenn die Engagierten der Überzeugung sind, dass ihr Einsatz den Nutznießer (Menschen, Kultur, Natur etc.) wirklich nützt und auch, wenn der besondere Charakter des freiwilligen Engagements gewahrt bleibt. Sie wollen ihre Fähigkeiten einsetzen können und selbst beim Engagement Kompetenzen und Bedeutung gewinnen. Sie legen Wert darauf, dass das Engagement gut mit ihrem Leben zu verbinden und, dass diese Arbeit von einer Institution oder der Kommune unterstützt und anerkannt wird. In den von uns untersuchten Bereichen achten die Engagierten genau darauf, ob die »nötigen Mittel« von den zuständigen öffentlichen Händen bereitgestellt werden. Diese hingegen können sich darauf verlassen, dass jeder Euro der dafür eingesetzten Mittel eine Wertschöpfung von sechs bis sieben Euro erbringt.

\section{Der Untersuchungsauftrag}

Ende 2007 beauftragte das Bayerische Sozialministerium für Arbeit und Sozialordnung, Familie und Frauen die stadt Würzburg. Cham, wie auch Würzburg, hat eine vergleichsweise gut entwickelte kommunale Unterstützungsstruktur Bürgerschaftlichen Engagements. Die zu untersuchenden Bereiche des Engagements waren:

- Freiwilligenagenturen, Koordinierungsstellen, Nachbarschaftshilfen

- Selbsthilfe

- Mütter- und Familienzentren

- Migration

- Senioren

- Jugendhilfe

- Freiwillige Feuerwehr

Andere ebenso wichtige Felder von Engagement wie beispielsweise die Kirchen oder der Sport waren nur insofern einbezogen, als doppelt Engagierte Erfahrungen aus diesen Bereichen mit einbrachten. Den ausgewählten sieben Feldern ist gemeinsam, dass sie nahe an kommunalen Pflichtaufgaben liegen.

\section{Die Kosten-Nutzen-Analyse}

Die Zahlen zur Ermittlung der Kosten und des Nutzens wurden mittels Fragebogen erhoben. Der Rücklauf lag, je 


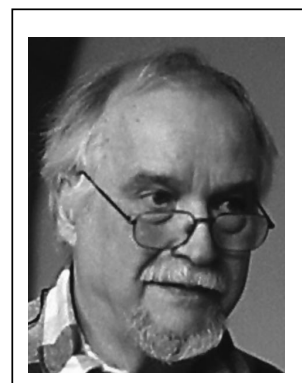

Dr. Walter Häcker ist Diplomphysiker; er war 15 Jahre lang Leiter der Volkshochschule Schorndorf und ist seit 20 Jahren freiberuflich als Organisationsentwickler und Moderator im Bereich des Bürgerschaftliche Engagements tätig. Als 2. Vorsitzender von foco e. V. vertritt er die Verbreitung des Community Organizing in Deutschland. Er engagiert sich international bei der Gründung des ECON (European Community Organizing Network) und lokal in der Region Stuttgart im »myself e. $V$. zur gegenseitigen Förderung am Arbeitsmarkt «, der "Stiftung + Arbeit « und der Zeitarbeitsgenossenschaft »ArbeitZuerst $«$.

E-Maildr.walter.haecker@web.de

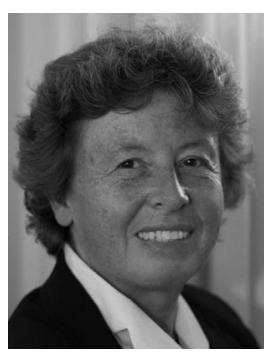

Doris Knaier ist Master of Social Work (MSW), Supervisorin (DGSV) und Diplom-Sozialpädagogin (FH). Sie arbeitet seit zehn Jahren freiberuflich als (Lehr-) Supervisorin, Lehrbeauftragte und Sozialwissenschaftlerin. Sie ist Projektleiterin der Infotage "Neues Wohnen, nachbarschaftlich leben « in bayerischen Mittelstädten (2008/2009) und arbeitete konzeptionell und redaktionell mit am bayerischen Wohnprojekt-Atlas, 1. und 2. Auflage (2005/2008). Sie arbeitet wissenschaftlich im Bereich Sozialplanung, gemeinschaftsorientiertes Wohnen, Wohnprojekte und Partizipation - letzteres auch im Kontext des Vereins Urbanes Wohnen e. V., Forum gemeinschaftlich Wohnen - Bundesvereinigung e. V. und Kompetenznetzwerk Wohnen.

Internet http://www.dorisknaier.de

nach Bereich, zwischen 36 und 70 Prozent. Für den Nutzen wurden die in einem Jahr durch bürgerschaftlich Engagierte in einer Einrichtung durchschnittlich geleisteten Stunden, multipliziert mit dem Stundenlohn, der für einen hauptamtlichen Mitarbeiter in diesem Bereich bezahlt würde. Sofern nicht tarifrechtlich ermittelbar, wurden (wie in anderen vergleichbaren Studien) acht Euro pro Stunde angesetzt. Bei Einsatz hauptamtlicher Kräfte wäre in aller Regel ein höherer Stundensatz zu bezahlen. Um nicht zu hoch zu rechnen, wurde in der Vergleichsrechnung dennoch dieser niedrige, nur knapp oberhalb des in der aktuellen Mindestlohndebatte liegende Satz, verwendet. In der Konsequenz liegt also die unmittelbare monetäre Wertschöpfung eher höher als hier angegeben. Um die Wertschöpfung zu erhalten, wurden von dem ermittelten Wert die Kosten für Aufwendungen (Personalund Sachkosten für Koordination, Räume, Verpflegung usw.) abgezogen (vgl. Abb. 1).

Das Verhältnis zwischen Kosten und Nutzen im Landkreis Cham und in der Stadt Würzburg ist insgesamt ver- gleichbar. In einigen Untersuchungsbereichen - beispielsweise Mütter- und Familienzentren, Selbsthilfe, Migration und Jugendhilfe - zeigen sich jedoch auffällige Unterschiede. Weitere Untersuchungen beispielsweise in Gebieten mit wenig entwickelter Unterstützungsstruktur, mit Bezug auf Bevölkerungs- und Bedarfszahlen oder unter Berücksichtigung aller Felder des Engagements, würden sicherlich noch wichtige Einblicke in gegebene Strukturen erlauben.

Im zweiten Teil des Fragebogens wurde nach den Folgen des Wegfalls des Engagements für die Einrichtung, die Zielgruppe, die Stadt, den Landkreis und die Engagierten selbst gefragt. Ziel war im Umkehrschluss die Bedeutung des Engagements zu ermessen. Das Ergebnis zeichnet ein düsteres Bild:

- Kosten explodieren, weil manche Tätigkeiten von Hauptamtlichen übernommen werden müssten oder ihr Wegfall erhöhte Aufwendungen beispielsweise im Gesundheitsbereich erfordern.

- Engagierte integrieren Menschen und setzen sich öffentlich für Menschen ein, die für sich selbst keine Lobby bilden können. Ohne Engagement verschwinden diese Menschen aus dem Blickfeld und das soziale Klima wird kalt und rau.

- Die soziale Landschaft verändert sich komplett. Ohne Engagement fallen Bereiche die Selbsthilfe oder Teile der sozialen und pflegerischen Altenarbeit weg.

\section{Die Nutzwertanalyse}

Die Methode »Nutzwertanalyse« wird eingesetzt bei vorrangig qualitativ zu bewertenden Entscheidungsproblemen in der Industrie oder bei ökologischen Vorhaben. Sie ergänzt die quantitative Evaluation durch qualitative Kriterien. Die Nutzwertanalyse ist eine formative Evaluation, bei der die wichtigste Rolle den Projekt-Teilnehmenden zufällt. Da die Beteiligten die Kriteriengewichtung und Kriterienbewertung im Dialog herbeiführen, erfolgt die Bewertung intersubjektiv (vgl. Abb. 4) Der konsensorientierte Dialog liefert hierfür wesentliche Erkenntnisse und Belege. Unsere Workshops in Cham und Würzburg verbanden die Erstellung des Gutachtens für die Beteiligten mit einem Lernprozess. So stellte sich neben den Ergebnissen ein weiterer Nutzen ein: Die in der Zusammenarbeit gewonnenen Erkenntnisse stießen bei den Teilnehmerinnen und Teilnehmern sofort Verbesserungen an im Engagement und in der Kooperation.

Die Nutzwertanalyse gewichtet im Konsens aller Beteiligten Kriterien des Bürgerschaftliches Engagements (Gewichtung) und wie gut diese erfüllt sind (Bewertung der Zielerreichung). Die Ziffer für die Wichtigkeit wird multipliziert mit der Ziffer für die Bewertung. Dies ergibt dann den Nutzwert, der als Maß für die relative Bedeutung des Kriteriums verstanden werden kann (vgl. Abb. 3).

Die Nutzwertanalysen wurden in zwei je zweitägigen, moderierten Konferenzen mit 32 Teilnehmenden in Cham und 48 Teilnehmende in Würzburg erstellt. Mitgemacht haben Engagierte und Nutznießer aus den genann- 


\begin{tabular}{|l|r|r|r|}
\hline Felder in Cham & BE-Nutzen & BE-Kosten & $\begin{array}{c}\text { Wert- } \\
\text { schöpfung }\end{array}$ \\
\hline $\begin{array}{l}\text { Freiwilligenagentur/ } \\
\text { Koordinierungsstellen }\end{array}$ & $483.832 €$ & $27.900 €$ & $455.932 €$ \\
\hline Selbsthilfe & $90.266 €$ & $32.770 €$ & $57.496 €$ \\
\hline $\begin{array}{l}\text { Mütter- und } \\
\text { Familienzentren }\end{array}$ & $10.480 €$ & $8.380 €$ & $2.100 €$ \\
\hline Migration & $36.300 €$ & $686 €$ & $35.614 €$ \\
\hline Senioren & $142.761 €$ & $10.380 €$ & $62.381 €$ \\
\hline Jugendhilfe & $1.616 .320 €$ & $177.868 €$ & $1.438 .452 €$ \\
\hline Freiwillige Feuerwehr & $2.452 .705 €$ & $338.704 €$ & $2.114 .001 €$ \\
\hline Gesamt & $80.720 €$ & $62.026 €$ \\
\hline
\end{tabular}

Abb. 1: Im Landkreis Cham in Ostbayern stiftet der Einsatz von einem Euro einen Nutzen von 7,24 Euro.

\begin{tabular}{|l|r|r|r|}
\hline Felder in Würzburg & BE-Nutzen & BE-Kosten & $\begin{array}{c}\text { Wert- } \\
\text { schöpfung }\end{array}$ \\
\hline $\begin{array}{l}\text { Freiwilligenagentur/ } \\
\text { Koordination/ } \\
\text { Nachbarschafts-Hilfe }\end{array}$ & $3.079 .392 €$ & $560.700 €$ & $2.518 .692 €$ \\
\hline Selbsthilfe & $1.420 .396 €$ & $47.120 €$ & $1.373 .276 €$ \\
\hline $\begin{array}{l}\text { Mütter- und } \\
\text { Familienzentren }\end{array}$ & $11.200 €$ & $5.500 €$ & $5.700 €$ \\
\hline Migration & $72.012 €$ & $12.300 €$ & $59.712 €$ \\
\hline Senioren & $779.510 €$ & $69.300 €$ & $710.210 €$ \\
\hline Jugendhilfe & $990.000 €$ & $300.000 €$ & $690.000 €$ \\
\hline Freiwillige Feuerwehr & $690.000 €$ & $6.744 .050 €$ & $1.057 .230 €$ \\
\hline Gesamt & $62.310 €$ & $329.230 €$ \\
\hline
\end{tabular}

Abb. 2: In der Stadt Würzburg stiftet der Einsatz von einem Euro einen Nutzen von 6,38 Euro

\section{Die Nutzwertanalyse}

Durch Gewichtung und Bewertung ergibt sich der Nutzen eines Projekts aus Sicht der Beteiligten:

Gewichtung x Bewertung

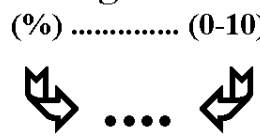

\section{$=$ Nutzwert}

Abb. 3: Bei der Nutzwertanalyse werden im Konsens aller Beteiligten die Kriterien gesucht und ihr Zielerreichungsgrad festgelegt.

ten Feldern, Vertreter von Institutionen, Verwaltung und Politik. Untersucht wurde in beiden Konferenzen nach den beteiligten Gruppen als Hauptkriterien:

- die Engagierten

- die Nutznießer: Menschen, Natur, Kultur

- die Institutionen, in deren Rahmen das Bürgerschaftliche Engagement angeboten wird

- Stadt, Gemeinde, Landkreis, der Staat

Den konkret ausformulierten Katalog von Haupt- und Unterkriterien wie auch der Erläuterungen und Beispiele sind im Internet zugänglich (1).

Die Ergebnisse in Cham (vgl. Abb. 4) weichen nicht grundsätzlich von diesen Ergebnissen aus Würzburg ab. Auch sie können im Internet eingesehen werden (1).

Die Abbildung 4 zeigt, mit welchem Gewicht im Konsens der Konferenz die einzelnen Haupt- und Unterkriterien gesehen und wie ihre Verwirklichung (auf einer Skala von 1 bis 10) bewertet wurden. So war beispielsweise das Unterkriterium 2.1 im Hauptkriterium Nutznießer: »2.1 Die Nutznießer bekommen im Ergebnis etwas Gutes« das Wichtigste, es ist mit einer Bewertung von 8 (von 10) noch ausbaufähig im Grad der Zielerreichung. Die Flächendarstellung macht die einzelnen Nutzwertziffern und ihre Relationen zueinander anschaulich.

\section{Schlussfolgerungen}

Warum ist es sinnvoll, in Politik, Verwaltung und Institutionen Bürgerschaftliches Engagement zu fördern? Welche Maßnahmen stärken das Engagement tatsächlich?

Mit am wichtigsten (Nutzwert 60,0; siehe Hauptkriterium 2 in Abb. 4) waren den Beteiligten die Fragen nach dem tatsächlichen Ergebnis des Engagements (»etwas Gutes «) und der Beziehung zwischen Engagiertem und Nutznießer. Hier liegt der Kern der Motivation. In den Workshops herrschte die Überzeugung »Wir tun Gutes«, aber auch die Frage »Ist das, was wir gut meinen auch wirklich gut?«

Fortbildungen, Supervision, Unterstützung von Hauptamtlichen bis hin zur Krisenintervention sichern, dass gutem Willen auch für die Nutznießer gute Taten folgen. Die Beziehung zwischen Engagiertem und Nutznießern darf nicht zur Fessel werden, gerade weil die Bereitschaft, mehr zu tun als geplant vorhanden ist. Engagierte suchen sich gerne ihr Tätigkeitsfeld selbst. Sie lassen sich sozusagen von der Aufgabe finden. Gesehen wird aber auch die Gefahr der »Quasi-Professionalisierung ohne Entlohnung «. Mit der Bewertung 8 ist die Zielerreichung hoch, doch noch ausbaufähig.

Fast gleich gewichtet (Nutzwert 56,3; Hauptkriterium 1) wurde der Nutzen, den die Engagierten selbst haben wollen: sinnvolle Arbeit machen, die eigenen Fähigkeiten einsetzen und Kompetenzen gewinnen. Der Wunsch nach persönlichem Wachstum ist ein wichtiges Motiv zu 


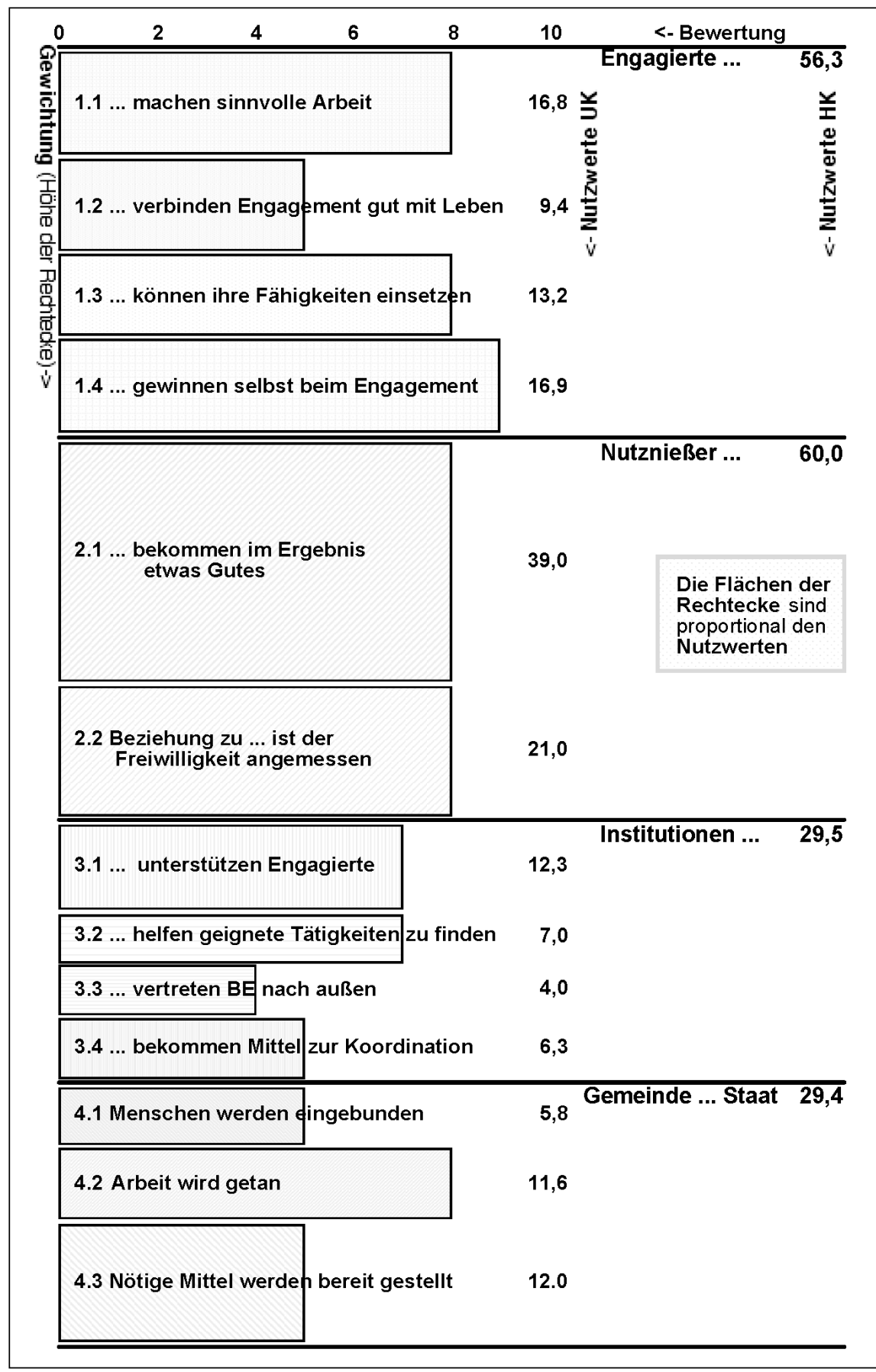

Abb. 4: Mit am wichtigsten waren nach der Nutzwertanalyse für die Stadt Würzburg den Beteiligten der tatsächliche Nutzen ihres Engagements (»Nutznießer bekommen im Ergebnis etwas Gutes «).

\section{Anmerkungen}

(1) Die Kurzfassung (20 Seiten; fertiggestellt Anfang Juni 2008) ist zu finden im Internet unter http://www.stmas.bayern.de/sozialpolitik/ehrenamt/wertgutachten.htm, die Langfassung (166 Seiten) im Internet unter http://www.wirfuer-uns.de/landesnetzwerk/gutachten_gkwh.pdf. Weitere, im Text nicht genannte, wissenschaftliche Mitarbeitende am Gutachten waren als MitProjektleiter Prof. Dr. Egon Endres, Christine Ginger, zuständig für die Kosten-Nutzen-Analyse und Erdenetungalag Byambajav, welche vergleichende Analysen der untersuchten Gebietskörperschaften beisteuerte.

(2) Vergleiche z. B. Ulich, Eberhard: Arbeitspsychologie, Schäffer-Poeschel 2005.
Engagement. Fortbildungen erweitern die Kompetenzen, Aufwandsentschädigungen ermöglichen jungen Menschen und materiell schlecht gestellten Bürgerinnen und Bürgern das Engagement. Die bayerische Ehrenamtsversicherung wurde in diesem Zusammenhang als ein wichtiger Schritt gesehen. Die Anrechnung auf die Rente wird gewünscht, steuerliche Anrechnungsmöglichkeiten sind zu wenig bekannt. Engagement soll sich auch beruflich lohnen, beispielsweise durch einen Nachweis bei Bewerbungen.

Die Institutionen (Nutzwert 29,5; Hauptkriterium 3) sollen das Engagement fördern, indem sie es öffentlich darstellen, Vernetzung und Erfahrungsaustausch im jeweiligen Feld und feldübergreifend im Stadtteil ermöglichen. Engagierte wollen neben dem zeitlichen Einsatz nicht auch noch eigene finanzielle Mittel einsetzen müssen. Internetunterstützung, Räume und Material fördern das Engagement. Hierzu benötigen die Institutionen, in deren Rahmen das Bürgerschaftliche Engagement angeboten wird, ihrerseits auch ausreichende öffentliche Förderung wie auch bessere Vertretung nach außen und in der Öffentlichkeit. Diese alles ist noch sehr ausbaufähig (Bewertung 5 und 4 von 10).

Die unterstützende Rolle der öffentlichen Hände (Nutzwert 29,4; Hauptkriterium 4) erfuhr mit die niedrigsten Bewertungen im Grad der Zielerreichung. Will man eine deutliche Verbesserung des Bürgerschaftlichen Engagements erreichen hat die Bereitstellung der nötigen Mittel durch die öffentlichen Hände Priorität. Die Beteiligten forderten wirkliche Teilhabe, statt nur allgemeine Beteiligung, politischen Entscheidungen an, die für die Durchführung des Engagements relevant sind.

Ein Ergebnis des Gutachtens ist: Effizient organisiertes und persönlich befriedigendes Engagement zeichnet sich durch sieben Merkmale aus, die auch in der Arbeitspsychologie als »Kriterien für gute Arbeit « (2) genannt werden: Ganzheitlichkeit, Variabilität, Autonomie und Handlungsspielraum, soziale Interaktion und Unterstützung, Lern- und Entwicklungspotenziale, Zeitelastizität und stressfreie Regulierbarkeit, Sinnhaftigkeit. 